\title{
Deep Investigation on Natural Convection Flow of a Couple Stress Fluid with Nanoparticles in an MHD Vertical Porous Channel with Convective Boundary Conditions
}

\author{
Mhamed Tayeb, Mohamed Najib Bouaziz* \\ Biomaterials and Transport Phenomena Laboratory, University of Medea, Medea 26000, Algeria
}

Corresponding Author Email: Bouaziz.mohamed@univ-medea.dz

https://doi.org/10.18280/ijht.380226

Received: 7 December 2018

Accepted: 11 April 2020

\section{Keywords:}

couple stress fluid, nanoparticle, MHD, natural convection, Soret and Dufour effects, heat and mass transfer

\begin{abstract}
This paper looks at the MHD natural convection flow through a vertical porous channel of couple stress fluid nanoparticles under convective heat conditions at the wall of the channel. Incorporated Soret and Dufour effects lead to strong coupled and highly nonlinear differential systems. To describe accurately all the physical phenomenon, the nanoparticle's volume fraction is taken into account. The useful technical of the similarity transformations is used to produce an ordinary system which is numerically solved. Graphical illustrations containing non-dimensional velocity, temperature, concentration and concentration of nanoparticles are extensively presented for different values of various thermophysical parameters. Main quantities of interest as Nusselt and Sherwood numbers at both the walls are tabulated.
\end{abstract}

\section{INTRODUCTION}

The flow of a non-Newtonian fluid with combined heat and mass transfer is frequently encountered in many engineering and industrial processes and it's increasingly used in several researches, recently, due to its wide range of applications like chemical engineering, environmental dynamics, cooling of electronic equipment, building ventilation and heat removal in nuclear technology.

Non-Newtonian fluids become very interesting for research because traditional Newtonian fluids cannot accurately describe the characteristics of actual promoted fluids, and their mechanics neglect the size effect of the material particles, which are included to improve the transfer rate of heat due to their higher thermal conductivity compared to the base fluid. The technical word 'nanofluid' was proposed for the first time by Choi and Eastman [1]. Nanofluids are used in many fields such as chillers, nuclear reactors, spacecraft, and heat exchangers to simulate the flow behavior of a lubricant with its own particles. Many theories of the micro-continuum have been generated, as the micropolar fluid model formulated by Eringen [2]. Heat transfer by nanofluid has been studied by many authors like Das et al. [3], and Das and Stephen [4], however Buongiorno [5] proposed an interesting mathematical nanofluid model based on the magnetic distribution effects in the absence of turbulent eddies, with Brownian diffusion and thermophoresis which are highlighted as the dominant slip mechanisms .

The couple stress fluid is a model of a non-Newtonian fluid whose theory is developed by Stokes $[6,7]$. He proposed the micron-continuum theory with polar effects and defined the rotation field in terms of velocity field to establish the constitutive relationship between stress and strain rate. Synthetic fluids, polymers, animal blood, thickened oils, synovial fluid in synovial joints and suspension fluids are the fields of application of the couple-stress fluids [8-15].

The incompressible flows through a porous pipe of a couple stress fluid have been studied recently and analyzed by several researchers [16-21]. The inertial effect should be significant at a higher rate and could be explained by the addition of a squared velocity term in the momentum equation, which is known as the Forchheimer extension of Darcy's law. A full detailed review of convective heat transfer, in Darcy and nonDarcy porous media, can be found in the book of Nield and Bejan [22]. Bujurke and Naduvinamani [23] studied the performance of a narrow porous bearing, lubricated with a couple stress fluid. Srinivasacharya and Kaladhar [24] based their studies on Hall and lon-slip effects for the mixed convection flow of the couple stress fluid between two parallel plates. Akhtar and Shah [25] presented an analytical solution for unsteady flows of couple stress fluid between two parallel plates, while Ramesh [26] conducted a study on the effects of slip and convection conditions on the peristaltic flow of the couple stress fluid in a porous asymmetric channel. Kaladhar and Srinivasacharya [27] directed numerical and experimental studies of natural convection between vertical flat plates with asymmetric heating.

Recently, Hayet et al. [28] examined Soret and Dufour effects with chemical reaction of peristaltic motion of the MHD couple stress fluid with the convective conditions of heat and mass transfer in an inclined asymmetric channel.

When heat and mass transfer occur simultaneously in a moving fluid, the relationships between flows and drive potentials are closer. It has been observed that a flow of energy can be generated, not only by temperature gradients, but also by concentration gradients. The energy flow caused by the concentration gradient is called the thermo-diffusion effect (Dufour). In most studies of the heat and mass transfer process, the Dufour effect is neglected because of its order of magnitude which is smaller than the effects described by Fourier and Fick laws, but it can be considered as a secondary phenomenon. Srinivasacharya and Kaladhar [29] studied Soret and Dufour effects with the convective conditions of heat and mass transfer of the couple stress fluid through a semi-infinite 
vertical plate. The same authors [30] studied the Soret and Dufour effects on steady free convective heat and mass transfer flow between two vertical parallel plates in couple stress fluid with chemical reaction. Eldabe et al. [31] examined the influence of Soret, Darcy, Hartmann and Schmidt numbers, and couple stress fluid parameter, on the heat and mass transfer with MHD flow of couple stress fluid through a porous medium. The irreversibility of heat and mass transfer of a couple stress nanofluid flow through a saturated magnetoporous vertical channel and a radiative heat transfer with entropy generation was realized in the papers [32,33]. Mondal et al. [34], presented a study of heat and mass transfer for a stress-induced nanofluid in a magneto-porous medium with heat generation and thermal radiation. Rauf et al. [35] considered unsteady mixed convective flow in the presence of nanoparticles of stress fluid, over an oscillatory stretching sheet, with heat absorption/generation effects.

In all the studies above, no literature has been reported about nanoparticles concentration which is the aim of our work in this article. We will study heat and mass transfer effect of a couple stress nanofluid convective flow through two parallel walls packed with a saturated magneto-porous medium. A boundary value problem is obtained and suitable technique is used to solve the nonlinear problem. Soret and Dufour, Darcy and Forchheimer effects, the couple stress parameter $S$, Hartmann number $M$, thermophoresis parameter $\mathrm{Nt}$, the Brownian motion parameter $N_{b}$ are studied. The influence of the parameters that characterize the flow are given numerically through graphs and discussed afterwards.

\section{MATHEMATICAL FORMULATION}

Consider the hydromagnetic steady laminar free convection flow of an incompressible, electrically and thermally conducting couple stress with nanoparticle between two vertical permeable parallel plates spaced $2 \mathrm{~d}$, in the presence of a uniform transverse magnetic field of strength $\mathrm{B}_{0}$ applied parallel to $y$-axis.

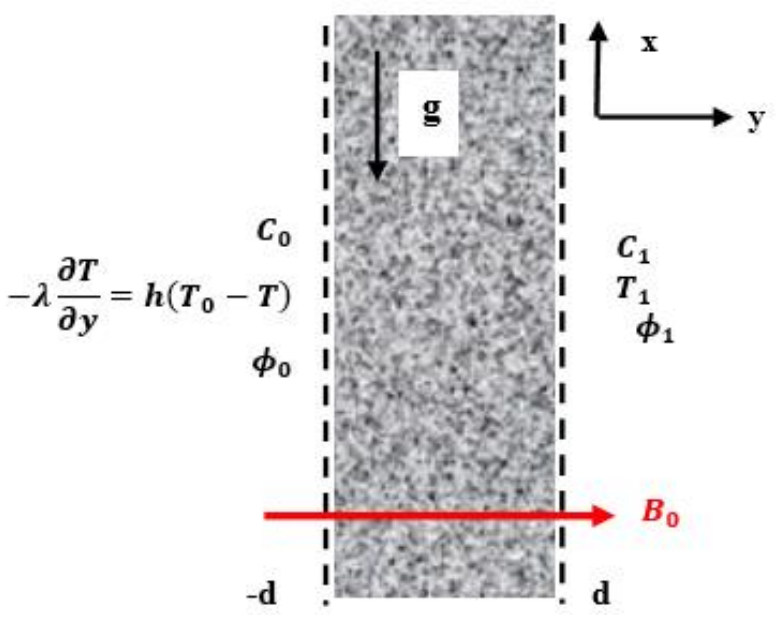

Figure 1. Physical configuration

The influences of external uniform magnetic fields of strength $\mathrm{B}_{0}$, transversally applied, are on the flow field. The effect of magnetic Reynolds number and the induced electric field are supposed to be minor and insignificant.

We assume that the left plate $(\mathrm{y}=-\mathrm{d}$ is convectively heated with hot fluid at temperature $T_{0}$ per heat transfer coefficient $h$, concentration $\mathrm{C}_{0}$ and concentration of nanoparticles $\phi_{0}$, while the right plate $(\mathrm{y}=\mathrm{d})$ is exposed to a uniform temperature $\mathrm{T}_{1}$ and a concentration $\mathrm{C}_{1}$ and the concentration of nanoparticles $\phi_{1}$. The physical quantities depend only on the coordinate $y$ because the boundaries in the $\mathrm{x}$ direction are of an infinite dimension, without loss of generality. The fluid properties are assumed constant whereas Dufour effect is taken into consideration. The physical model of the problem is presented in Figure 1.

With the above assumptions and Boussinesq approximations of energy and concentration, the equations governing the steady flow of an incompressible couple stress fluid are:

\section{Continuity Equation:}

$$
\frac{\partial u}{\partial x}+\frac{\partial v}{\partial y}=0
$$

\section{Momentum Equations:}

$$
\begin{gathered}
-v_{0} \frac{\partial u}{\partial y}=-\frac{1}{\rho_{f}} \frac{\partial P}{\partial x}+\frac{\mu}{\rho_{f}} \frac{\partial^{2} u}{\partial y^{2}}-\frac{\eta}{\rho_{f}} \frac{\partial^{4} u}{\partial y^{4}}-\frac{\sigma B_{0}^{2} u}{\rho_{f}}-\frac{\mu}{\rho_{f} K} u- \\
-\frac{c_{f}}{\sqrt{K}} u^{2}+\left(1-\phi_{1}\right) g\left(\beta_{T}\left(T-T_{1}\right)+\beta_{C}\left(C-C_{1}\right)\right)- \\
-g\left(\left(\frac{\rho_{p}-\rho_{f}}{\rho_{f}}\right)\left(\phi-\phi_{1}\right)\right)
\end{gathered}
$$

\section{Energy Equation:}

$$
\begin{gathered}
-v_{0} \frac{\partial T}{\partial y}=\frac{\lambda}{\left(\rho C_{P}\right)_{f}} \frac{\partial^{2} T}{\partial y^{2}}+\frac{2 \mu}{\left(\rho C_{P}\right)_{f}}\left(\frac{\partial u}{\partial y}\right)^{2}+\frac{\eta}{\left(\rho C_{P}\right)_{f}}\left(\frac{\partial^{2} u}{\partial y^{2}}\right)^{2} \\
+\varepsilon \frac{\left(\rho C_{P}\right)_{p}}{\left(\rho C_{P}\right)_{f}}\left[D_{B} \frac{\partial \phi}{\partial y} \frac{\partial T}{\partial y}+\frac{D_{T}}{T_{m}}\left(\frac{\partial T}{\partial y}\right)^{2}\right]+\frac{D_{m} K_{T}}{C_{S} C_{P_{f}}} \frac{\partial^{2} C}{\partial y^{2}}+\frac{\sigma B_{0}^{2} u^{2}}{\left(\rho C_{P}\right)_{f}}
\end{gathered}
$$

\section{Concentration Equation:}

$$
-\frac{1}{\varepsilon} v_{0} \frac{\partial C}{\partial y}=D_{m} \frac{\partial^{2} C}{\partial y^{2}}+\frac{D_{m} K_{T}}{T_{m}} \frac{\partial^{2} T}{\partial y^{2}}
$$

\section{Concentration of nanoparticles Equation:}

$$
-\frac{1}{\varepsilon} v_{0} \frac{\partial \phi}{\partial y}=D_{B} \frac{\partial^{2} \phi}{\partial y^{2}}+\frac{D_{T}}{T_{m}} \frac{\partial^{2} T}{\partial y^{2}}
$$

The boundary conditions are:

$$
\begin{gathered}
u=0 \text { at } y= \pm d \\
u_{y y}=0 \text { at } y= \pm d \\
-\lambda \frac{\partial T}{\partial y}=h_{f}\left(T_{0}-T\right), C=C_{0}, \phi=\phi_{0} \text { at } y=-d \\
T=T_{1}, C=C_{1}, \phi=\phi_{1} \text { at } y=d
\end{gathered}
$$

The boundary condition (6) corresponds to the classical noslip condition from viscous fluid dynamics. The boundary condition (7) implies that the couple stresses are zero at the plate surfaces. The boundary condition (8) and (9) corresponds the temperature, concentration and concentration of nanoparticles on the surfaces of the plate $(-d)$ and $(+d)$ 
respectively.

where, $u$ is the velocity component along $\mathrm{x}$ direction, $\mathrm{T}, \mathrm{C}$ and $\phi$ represent the temperature, concentration and nanoparticles concentration respectively, $v_{0}$ is the fluid velocity, $\mathrm{P}$ defines the pressure, $\mu$ signifies the coefficient of viscosity, $\rho_{f}$ and $\rho_{P}$ mean the density base liquid and density of nanoparticle respectively, $\eta$ denotes the couple stress viscosity parameter, $\beta_{T}$ and $\beta_{C}$ are the thermal expansion coefficient and the concentration expansion coefficient respectively, $\mathrm{g}$ is the acceleration due to gravity, $\sigma$ is the electrical conductivity, $B_{0}$ denotes the magnetic field, $\mathrm{K}$ designates permeability of porous medium (hydraulic conductivity), $\mathrm{c}_{\mathrm{f}}$ is the Forchheimer constant, $\lambda$ is the thermal conductivity of the fluid, $\left(\rho C_{P}\right)_{f}$ and $\left(\rho C_{P}\right)_{P}$ are the heat capacity at constant pressure of fluid and the heat capacity of nanoparticles respectively, $\alpha=\lambda /\left(\rho C_{P}\right)_{f}$ represents the thermal diffusivity, $\varepsilon$ is the porosity, $\mathrm{D}_{\mathrm{B}}$ indicates the Brownian diffusion coefficient, in $\mathrm{D}_{\mathrm{T}}=\mathrm{D}_{\mathrm{m}} \mathrm{K}_{\mathrm{T}}, \mathrm{D}_{\mathrm{T}}$ is the thermophoresis diffusion coefficient, $\mathrm{D}_{\mathrm{m}}$ is the molecular solutal diffusivity, $K_{T}$ indicates the thermal diffusion ratio, $\mathrm{T}_{\mathrm{m}}$ is the mean temperature, $\mathrm{C}_{S}$ is the concentration susceptibility. $\mathrm{h}_{\mathrm{f}}$ is the heat transfer coefficient between the plate and the hot fluid.

Introducing the following similarity transformations:

$$
\left\{\begin{array}{c}
\eta=\frac{y}{a}, X=\frac{x}{a}, u=\frac{v_{f}}{a} f, \theta=\frac{T-T_{1}}{T_{0}-T_{1}}, \\
\varphi=\frac{C-C_{1}}{C_{0}-C_{1}}, \psi=\frac{\phi-\phi_{1}}{\phi_{0}-\phi_{1}}
\end{array}\right.
$$

After using these transformations, we obtain the following nonlinear system of the differential equations:

$$
\begin{gathered}
A+\operatorname{Re} f^{\prime}+f^{\prime \prime}-S^{2} f^{(4)}+\mathrm{Gr}_{\mathrm{T}} \theta+\mathrm{Gr}_{\mathrm{M}} \varphi- \\
\mathrm{N}_{\mathrm{r}} \mathrm{Gr}_{\mathrm{T}} \psi-M f-D a \cdot f-F o . f^{2}=0 \\
\theta^{\prime \prime}+\operatorname{Re} \operatorname{Pr} \theta^{\prime}+2 E c \operatorname{Pr} f^{2}+E c \operatorname{Pr} S^{2} f^{\prime \prime 2}+ \\
+N b \theta^{\prime} \psi^{\prime}+N t \theta^{2}+D_{f} \operatorname{Pr} \varphi^{\prime \prime}+E c \operatorname{Pr} M f^{2}=0 \\
\varphi^{\prime \prime}+\operatorname{Re} \operatorname{Pr} \operatorname{Le}_{n} \varphi^{\prime}+S_{r} S c / \operatorname{Pr} \theta^{\prime \prime}=0 \\
\psi^{\prime \prime}+\operatorname{RePr}_{n} \psi^{\prime}+N t / N b \theta^{\prime \prime}=0
\end{gathered}
$$

where, primes denote the differentiation with respect to $\eta$ alone:

$A=-\frac{\partial \bar{P}}{\partial X} ;$ (the pressure gradient); where $\bar{P}=\left(\frac{a^{2}}{\rho_{f} v_{f}^{2}}\right) P$.

$R e=\frac{v_{0} a}{v_{f}} ;$ (the Reynolds number).

$S^{2}=\frac{\eta}{\rho_{f} a^{2} v_{f}} ;$ (the couple stress parameter).

$G r_{T}=\frac{\left(1-\phi_{1}\right) g \beta_{T}\left(T_{0}-T_{1}\right) a^{3}}{v_{f}{ }^{2}} ;$ (The thermal Grashof number)

$G r_{M}=\frac{\left(1-\phi_{1}\right) g \beta_{C}\left(C_{0}-C_{1}\right) a^{3}}{v_{f}^{2}} ;$ (The concentration Grashof number).

$N r=\frac{\left(\rho_{p}-\rho_{f}\right)\left(\phi_{0}-\phi_{1}\right)}{\rho_{f}\left(1-\phi_{1}\right) \beta_{T}\left(T_{0}-T_{1}\right)} ; \quad$ The nanofluid buoyancy ratio parameter).

$M=\frac{\sigma B_{0}^{2} a^{2}}{\rho_{f} v_{f}} ;$ (The magnetic field parameter)

$D a=\frac{a^{2}}{K} ;$ (Darcy number).
$F_{O}=\frac{c_{f} a}{\sqrt{K}} ;$ (Forchheimer number).

$\operatorname{Pr}=\frac{\left(\rho C_{P}\right)_{f} v_{f}}{\lambda} ;$ (The Prandtl number).

$E c=\frac{v_{f}{ }^{2}}{\left(\rho C_{P}\right)_{f} a^{2}\left(T_{0}-T_{1}\right)} ;$ (The Eckert number).

$N b=\frac{\left(\rho C_{P}\right)_{p}}{\left(\rho C_{P}\right)_{f}} \cdot \frac{\varepsilon D_{B}\left(\phi_{0}-\phi_{1}\right)}{\alpha_{f}}$; (The Brownian motion parameter)

$N t=\frac{\left(\rho C_{P}\right)_{p}}{\left(\rho C_{P}\right)_{f}} \cdot \frac{\varepsilon D_{T}\left(T_{0}-T_{1}\right)}{T_{m} \alpha_{f}} ;$ (The thermophoresis parameter).

With $\alpha_{f}=\frac{\lambda}{\left(\rho C_{P}\right)_{f}}$; (The thermal diffusivity).

$D_{f}=\frac{D_{m} K_{T}\left(C_{0}-C_{1}\right)}{v_{f} C_{S} C_{P f}\left(T_{0}-T_{1}\right)} ;$ (the Dufour number).

$S_{r}=\frac{D_{m} K_{T}\left(T_{0}-T_{1}\right)}{\left(C_{0}-C_{1}\right) T_{m} \alpha_{f}} ;$ (the Soret Number).

$L e_{n}=\frac{\alpha_{f}}{\varepsilon D_{m}} ;$ (Thermo-nanofluid Lewis number).

$\operatorname{Pr}_{n}=\frac{v_{f}}{\varepsilon D_{B}} ;$ (The Prandtl nanofluid number).

$H=\frac{h_{f} a}{\lambda}$; (The Biot number).

The boundaries conditions (6)-(9) in terms of velocity $f(\eta)$, temperature $\theta(\eta)$, concentration $\varphi(\eta)$ and concentration of nano-particles $\psi(\eta)$ become:

$$
\begin{aligned}
& f=0 ; f^{\prime \prime}=0 ; \theta^{\prime}=-H \theta ; \varphi=1, \psi=1 \text { at } \eta=0 \\
& f=0 ; f^{\prime \prime}=0 ; \theta=1 ; \varphi=0, \quad \psi=1 \text { at } \eta=1
\end{aligned}
$$

The physical quantities of interest are the local Nusselt and the local Sherwood numbers, which are defined as:

$$
N u=\frac{a q_{h}}{h\left(T_{0}-T_{1}\right)}, S h=\frac{a q_{m}}{D_{m}\left(C_{0}-C_{1}\right)}
$$

where, $\mathrm{q}_{\mathrm{h}}$ denotes heat flux and $\mathrm{q}_{\mathrm{m}}$ is the mass flux near the surface.

$$
q_{h}=-h\left(\frac{\partial T}{\partial y}\right)_{y=0}, q_{m}=-D_{m}\left(\frac{\partial C}{\partial y}\right)_{y=0}
$$

We obtain dimensionless version of these key design quantities:

$$
N u_{1,2}=-\left.\theta^{\prime}(\eta)\right|_{\eta=0,1}, S h_{1,2}=-\left.\varphi^{\prime}(\eta)\right|_{\eta=0,1}
$$

\section{RESULTS AND DISCUSSION}

The numerical and computational results are discussed through the graphical illustrations. The variation of Velocity, Temperature, Concentration and Concentration of nanoparticles for different values $\mathrm{S}_{\mathrm{r}}$ and $\mathrm{D}_{\mathrm{f}}$ numbers, $D a$ and $\mathrm{F}_{\mathrm{o}}$ numbers, as well as $\mathrm{S}, \mathrm{M}, \mathrm{Nt}, \mathrm{Nb}, G r_{T}, G r_{M}, L e_{n}, \mathrm{Nr}$ and $\mathrm{H}$, are studied and discussed through Figures [3-30]. The values used in the calculus are mentioned in each figure. Soret and Dufour numbers values are taken such as their product stay constant, meaning that the temperature $\mathrm{T}_{\mathrm{m}}$ is kept stable [34]. Mathematical software is used to find out the numerical results.

In absence of the nanoparticles $N t=N b=P r_{n}=N r=0$, and the model becomes classical couple stress fluid. For comparison, we put $D a=F_{O}=0$ as without porous medium, the magnetic field $\mathrm{M}=0$, the convective boundary conditions $H=0$ and the pressure gradient is taking $\mathrm{A}=0$. It's noted that $\mathrm{Br}=E c$. Pr, then the flow-field profiles, generated 
by the computer, of this study, are nearly similar with those of the results presented by Srinivasacharya and Kaladhar [29].

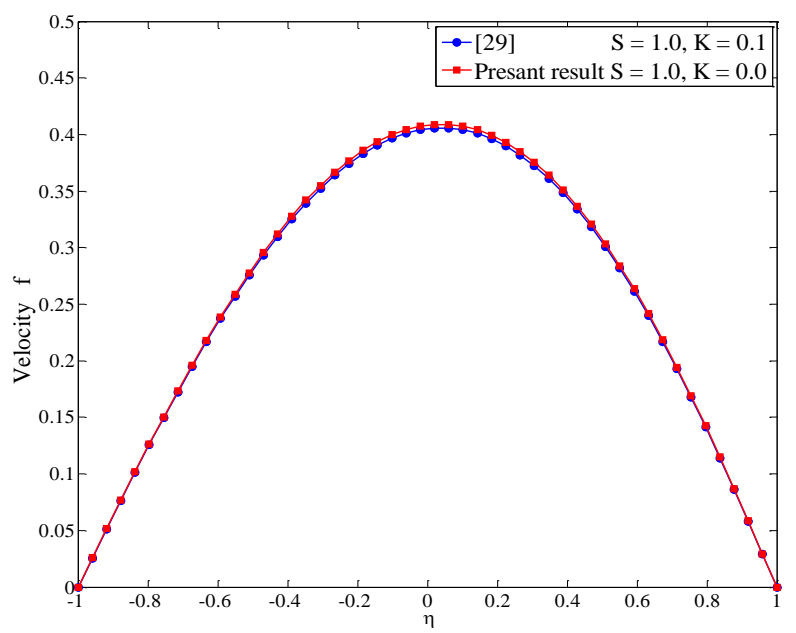

Figure 2. Comparison of velocity profile for the present result with Srinivasacharya's and Kaladhar [29] results

We have compared the present model to that of Srinivasacharya and Kaladhar [29] for two values of the couple stress parameter and for non-dimensional velocity. They are illustrated in Figure 2. The outcomes are found to be in good agreement. It can be observed from this figure that the two curves are almost identical with a small difference, that's because the results that we compared our study with, contain a chemical reaction which is absent in the reference. These results confirm the validity of the current numerical scheme used.

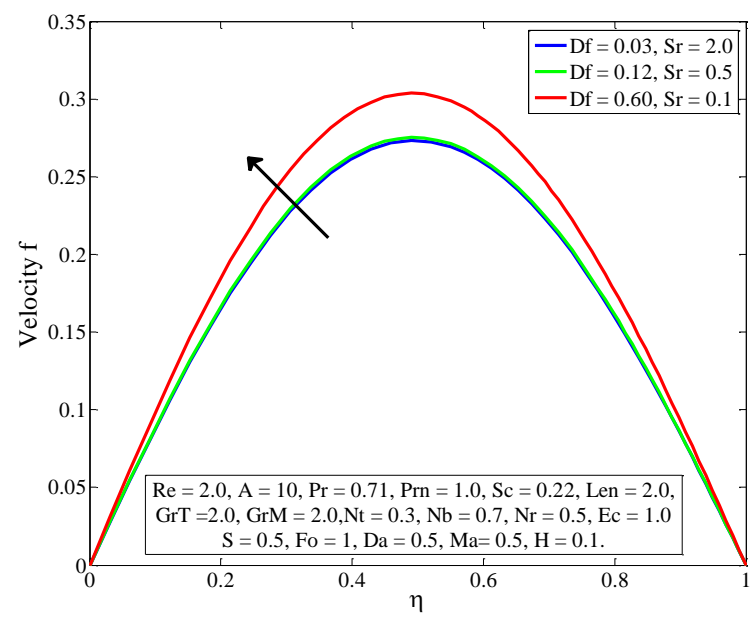

Figure 3. Velocity profile for different values of $D_{f}$ and $S_{r}$

The Figure 3 demonstrates the non-dimensional velocity $f(\eta)$ for different values of the Soret $\mathrm{S}_{\mathrm{r}}$ and Dufour $\mathrm{D}_{\mathrm{f}}$ numbers. It can be observed from this figure that the fluid velocity increases with the increase of Dufour number $\mathrm{D}_{\mathrm{f}}$ (or the decrease the Soret number $S_{\mathrm{r}}$ ).

The non-dimensional temperature $\theta(\eta)$ for different values of $S_{r}$ and $D_{f}$ numbers is presented in the Figure 4. Here the increase of the fluid temperature leads to the rise of $D_{f}$ (or the decrease of $\mathrm{S}_{\mathrm{r}}$ ). Strong thermodiffusion affect $f$ and more in $T$.

Figure 5 shows the non-dimensional concentration $\phi(\eta)$ for different values of $S_{r}$ and $D_{f}$ numbers. From this figure, it can be seen that the fluid concentration diminishes with the increase of $\mathrm{D}_{\mathrm{f}}$ (or the decrease of number $\mathrm{S}_{\mathrm{r}}$ ).

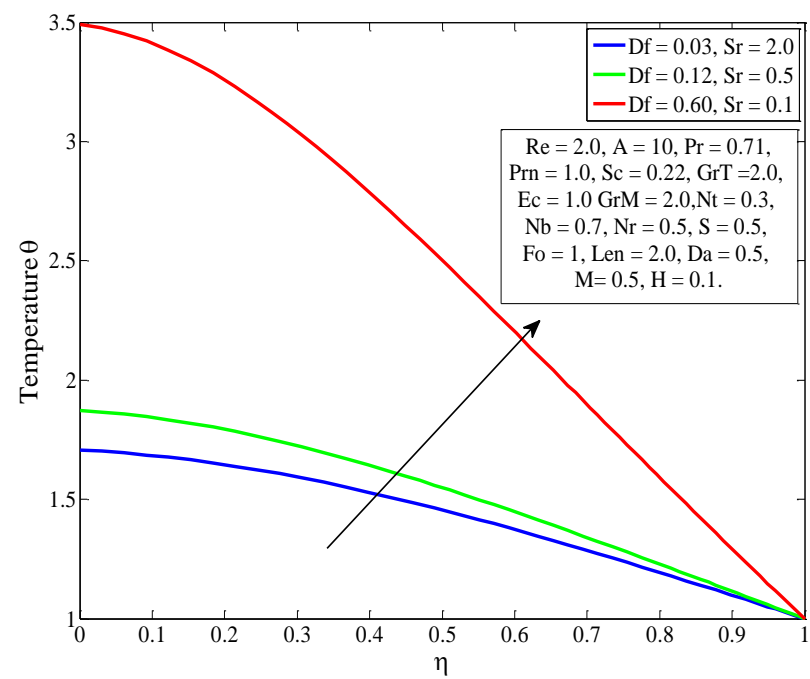

Figure 4. Temperature profile for different values of $D_{f}$ and $\mathrm{S}_{\mathrm{r}}$

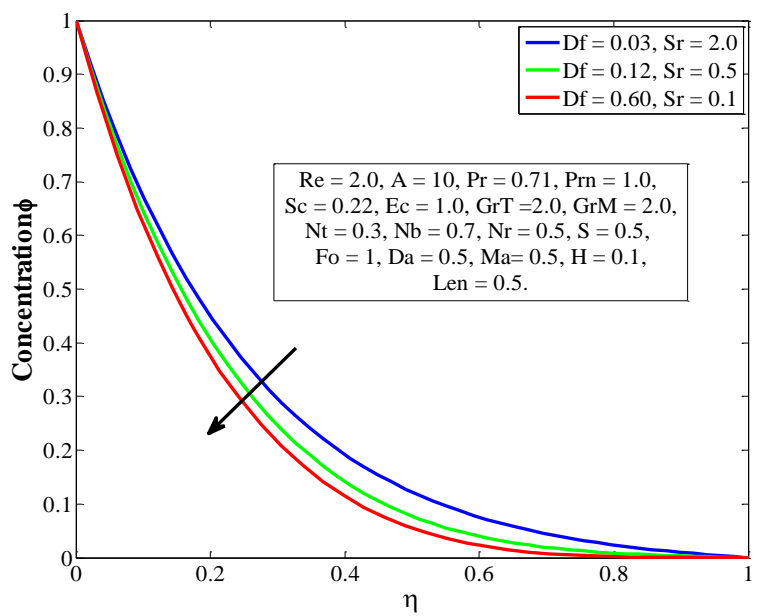

Figure 5. Concentration profile for different values of $D_{f}$ and $\mathrm{S}_{\mathrm{r}}$

Figure 6 represents the non-dimensional concentration of nanoparticles $\psi(\eta)$ for different values of $S_{\mathrm{r}}$ and $D_{\mathrm{f}}$ numbers. It's observed that the decrease of the nanoparticle's fluid concentration is caused by the augmentation of $\mathrm{D}_{\mathrm{f}}$ (or the deceleration of $\mathrm{S}_{\mathrm{r}}$ ).

Figure 7 shows the effects of Darcy $D a$ and Fo numbers on the velocity profile. As it is demonstrated; the velocity profile decelerates with increasing of Darcy number; meanwhile, the rise in Forchheimer number reduces the velocity profile. Also, as Darcy and Forchheimer numbers decrease, the temperature field declines, such it is illustrated on the Figure 8.

The Figure 9 expresses the concentration profile for different values of $D a$ and $F o$ numbers. It can be seen that their augmentation causes a little increase in the concentration profile, which expressed that no effect of structural medium on this quantity. The same effect is observed in Figure 10 which represents the non-dimensional concentration of nanoparticles.

In the Figures 11 to 14 , the effects of couple stress parameter $\mathrm{S}$ and Hartmann number $\mathrm{M}$ on the velocity, temperature, concentration and concentration nanoparticles non-dimensional profiles are presented. 


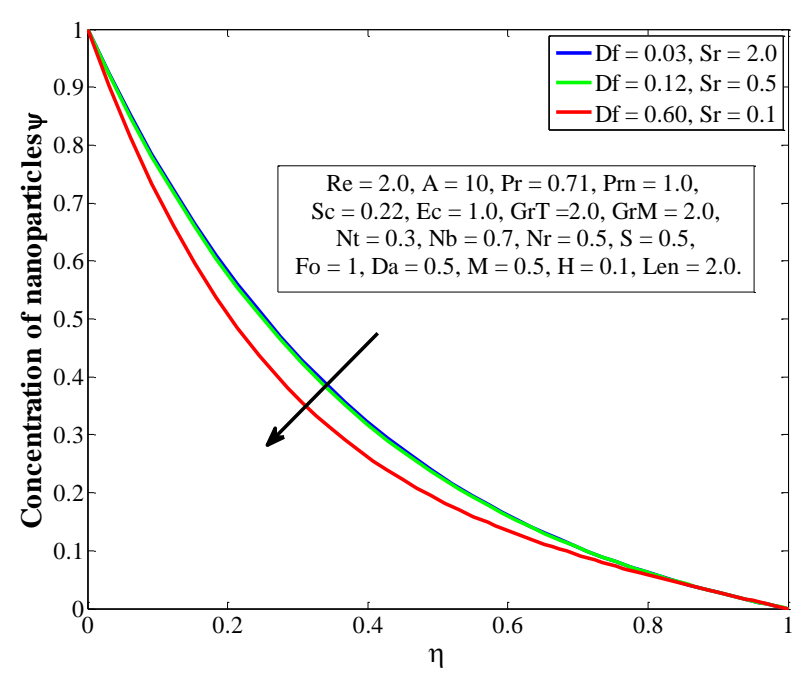

Figure 6. Concentration of nanoparticles profile for different values of $D_{f}$ and $S_{r}$

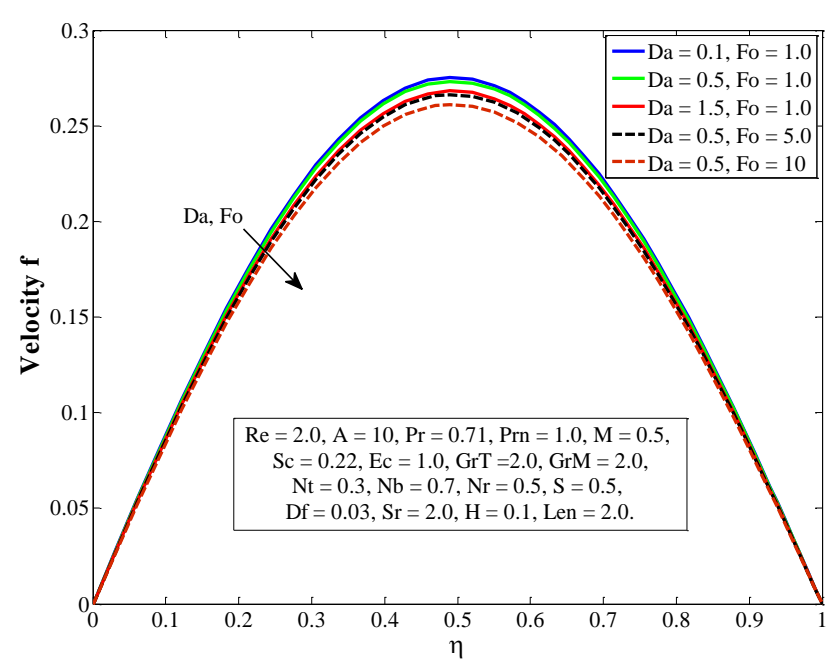

Figure 7. Velocity profile for different values of $\mathrm{Da}$ and $\mathrm{Fo}$

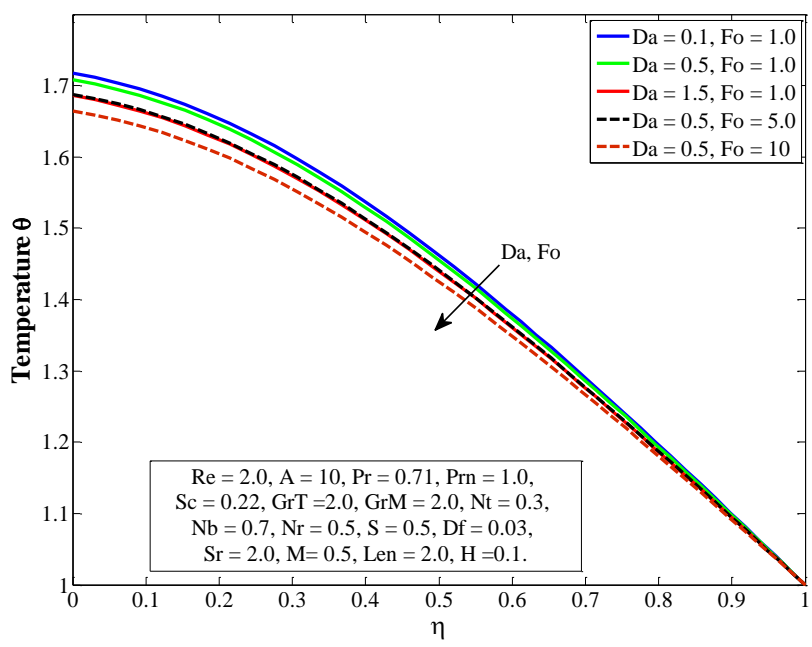

Figure 8. Temperature profile for different values of $D a$ and $F_{O}$

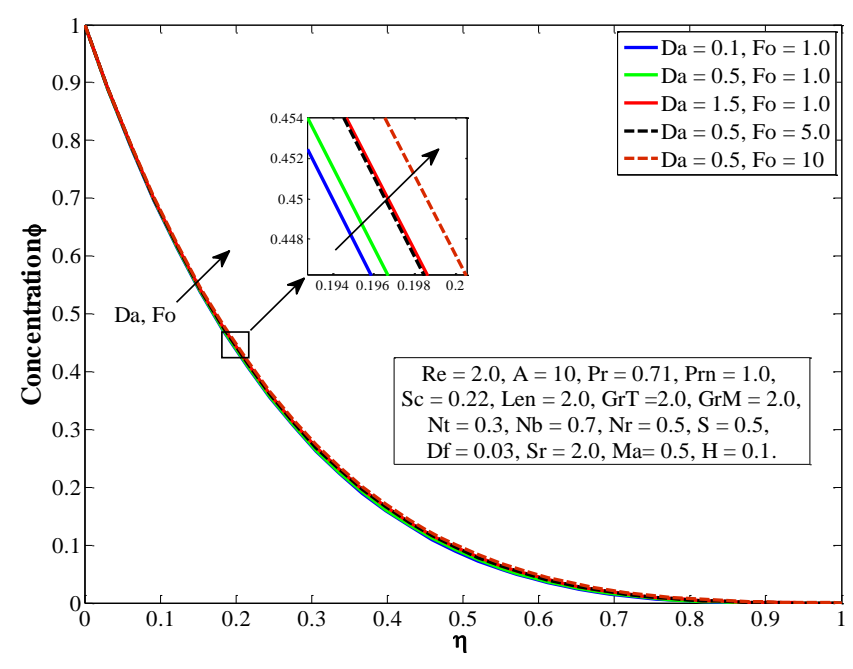

Figure 9. Concentration profile for different values of $D a$ and $\mathrm{Fo}$

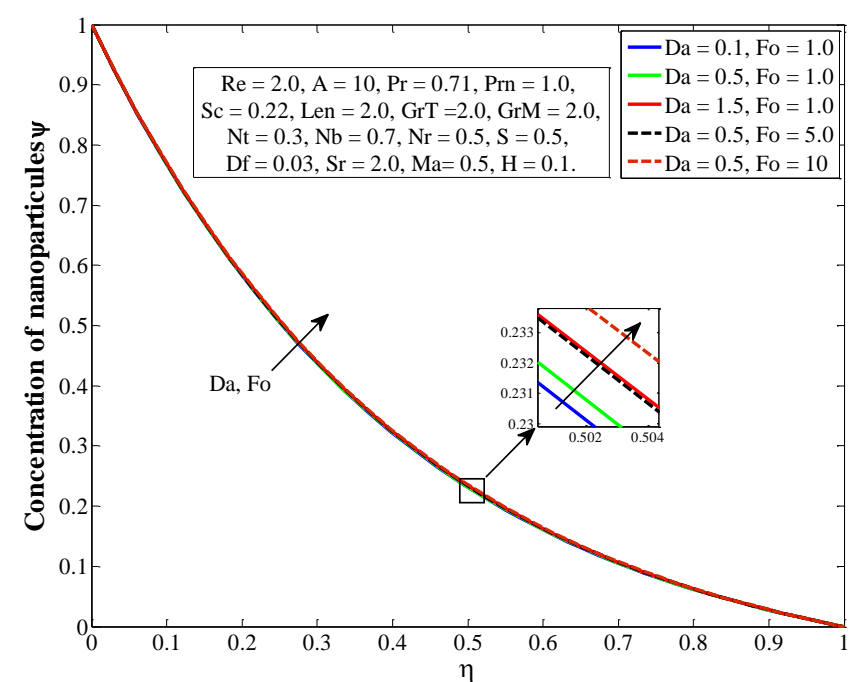

Figure 10. Concentration of nanoparticles profile for different values of $D a$ and $F o$

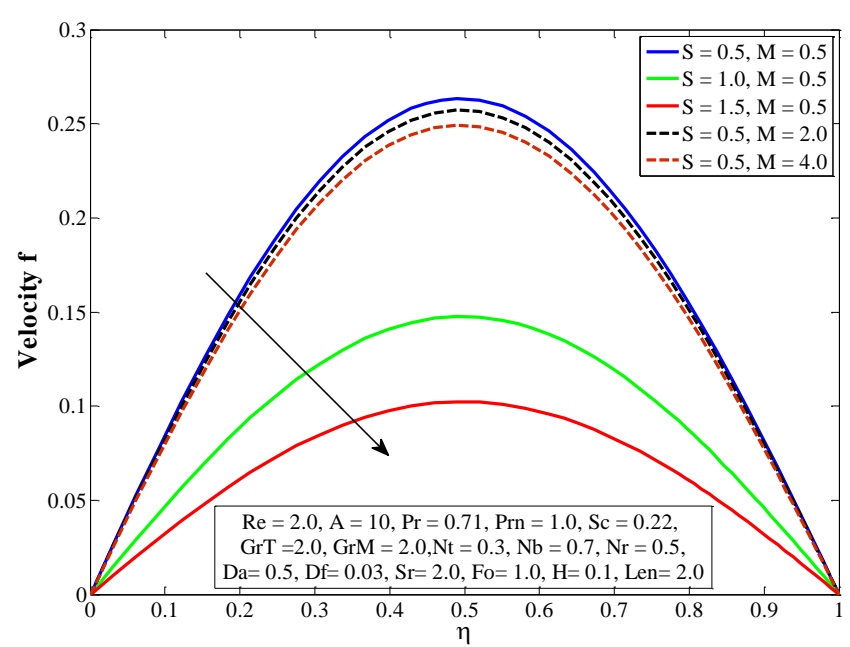

Figure 11. Velocity profile for different values of S and M 
It can be observed from Figure 11 that the velocity decreases as $\mathrm{S}$ and $\mathrm{M}$ increase. In the Figure 12, it can be shown that the temperature decreases also with the increase of couple stress parameter S or number Hartmann number $\mathrm{M}$.

Nevertheless, we observe from the Figures 13 and 14, that the raise of $S$ and $M$ cause a little increase in both of the concentration and the concentration of nanoparticles.

The Figures 15, 16, 17 and 18 demonstrate the effects of thermophoresis $\mathrm{Nt}$ and Brownian motion $\mathrm{Nb}$ parameters on the velocity $f(\eta)$, the temperature $\theta(\eta)$, the concentration $\phi(\eta)$ and the concentration of nanoparticles $\psi(\eta)$. It can be clearly observed that as $N t$ and $N b$ increase, both the velocity and the temperature augment, however the concentration decreases. Moreover, it's shown that as Nt increases, the concentration of nanoparticles profile decreases, and that the concentration of nanoparticles augments when $\mathrm{Nb}$ decelerates.

The Figures, from 19 to 22, represent the effects of the thermal Grashof number $G r_{T}$ and concentration Grashof number $G r_{M}$ on the non-dimensional velocity, the temperature, the concentration and concentration of the nanoparticle's profiles. It is noticed that the raise in each of these parameters cause the augmentation of velocity and temperature profiles.

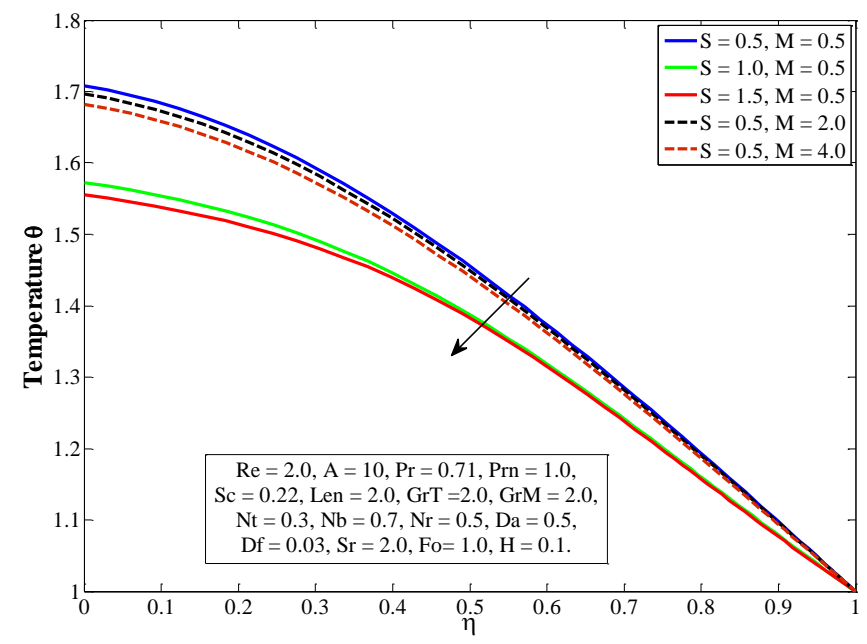

Figure 12. Temperature profile for different values of $S$ and $\mathrm{M}$

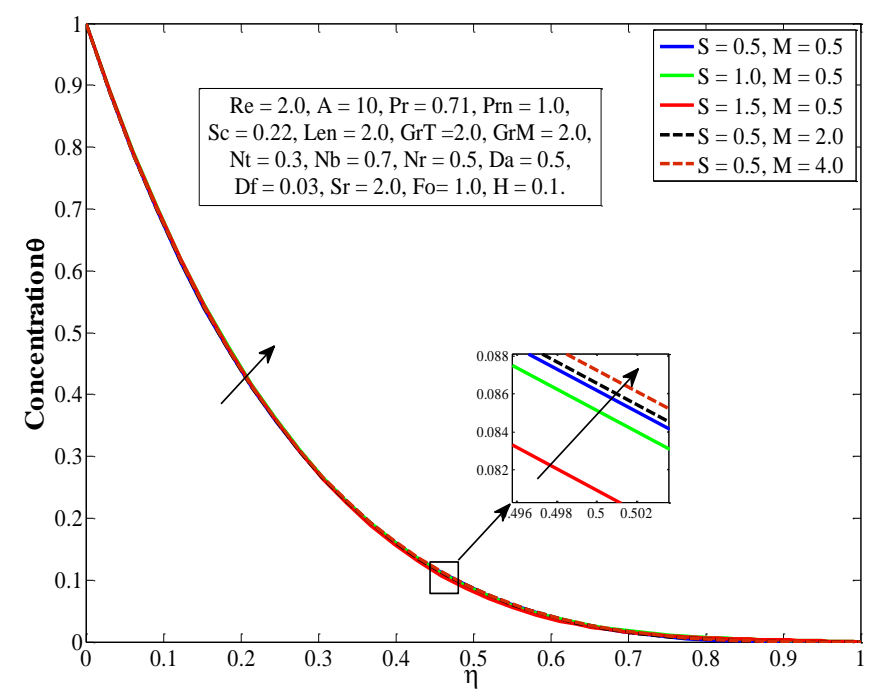

Figure 13. Concentration profile for different values of $S$ and $\mathrm{M}$

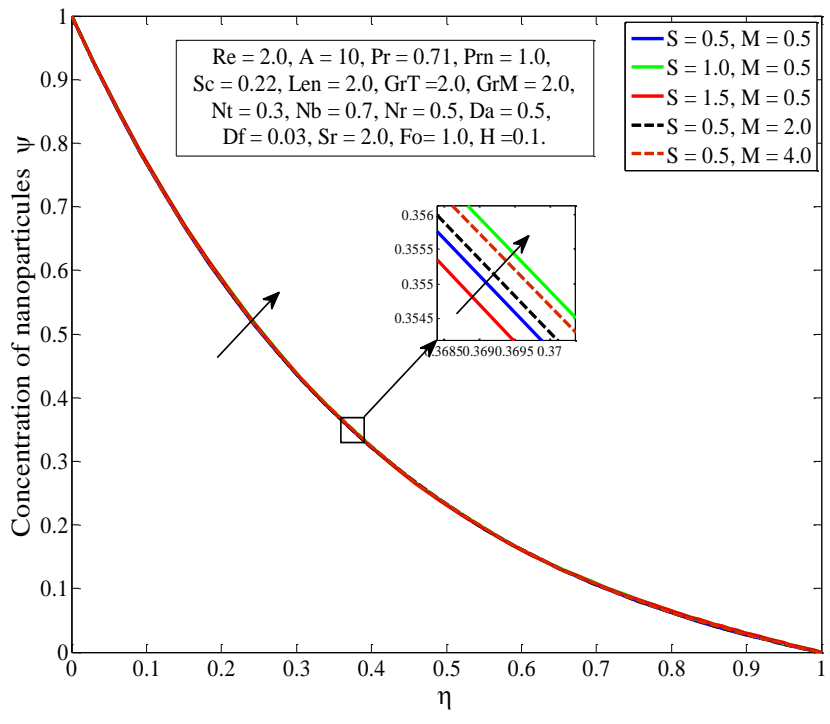

Figure 14. Concentration of nanoparticles profile for different values of $\mathrm{S}$ and $\mathrm{M}$

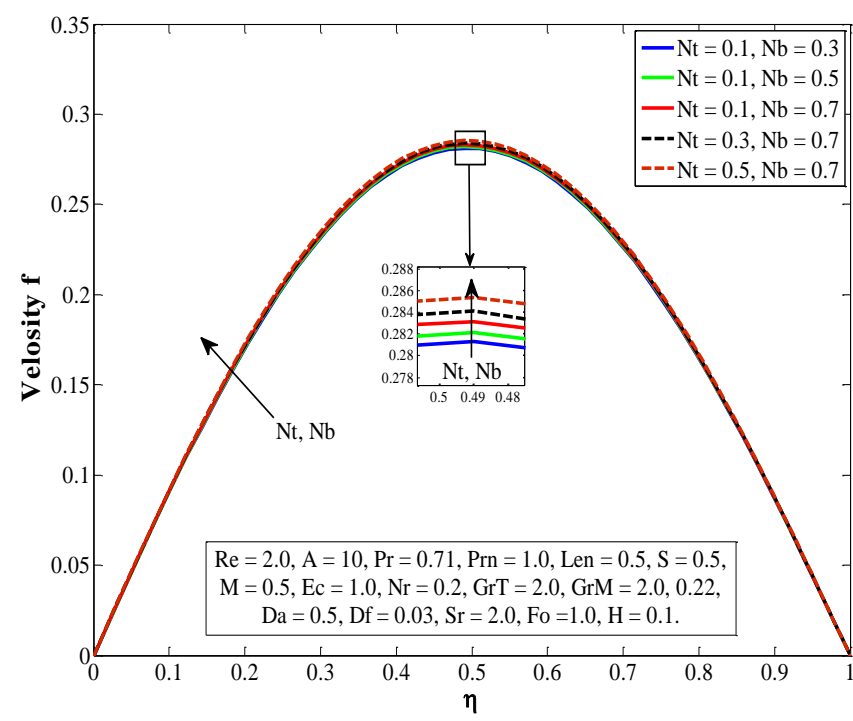

Figure 15. Velocity profile for different values of $\mathrm{Nt}$ and $\mathrm{Nb}$

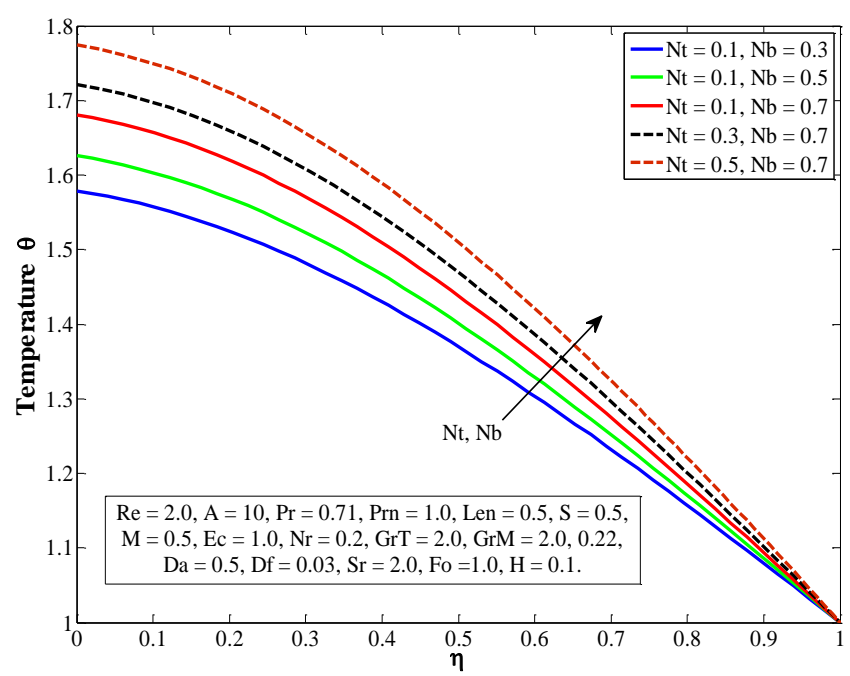

Figure 16. Temperature profile for different values of $\mathrm{Nt}$ and $\mathrm{Nb}$ 


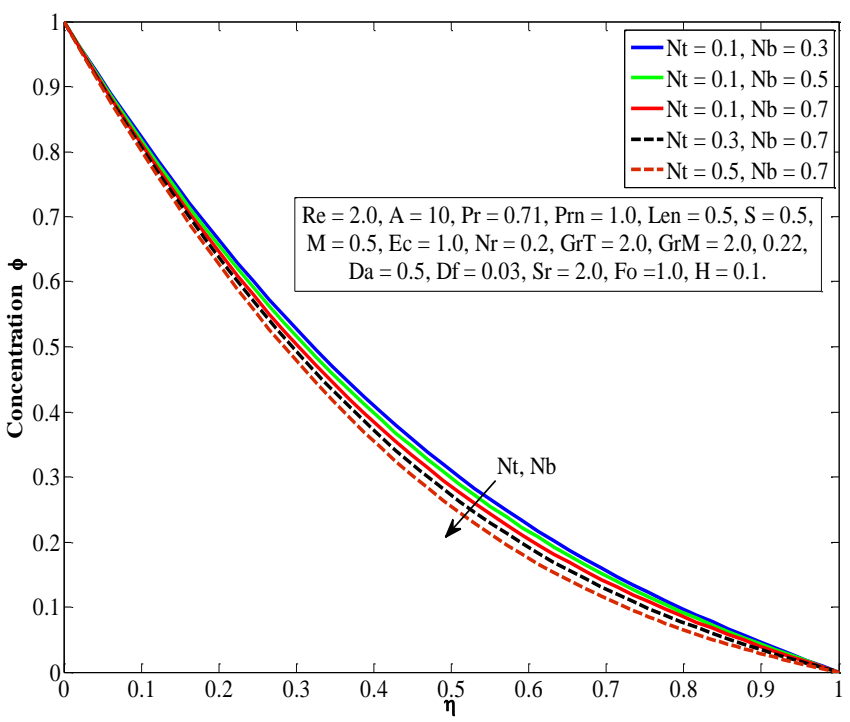

Figure 17. Concentration profile for different values of $\mathrm{Nt}$ and $\mathrm{Nb}$

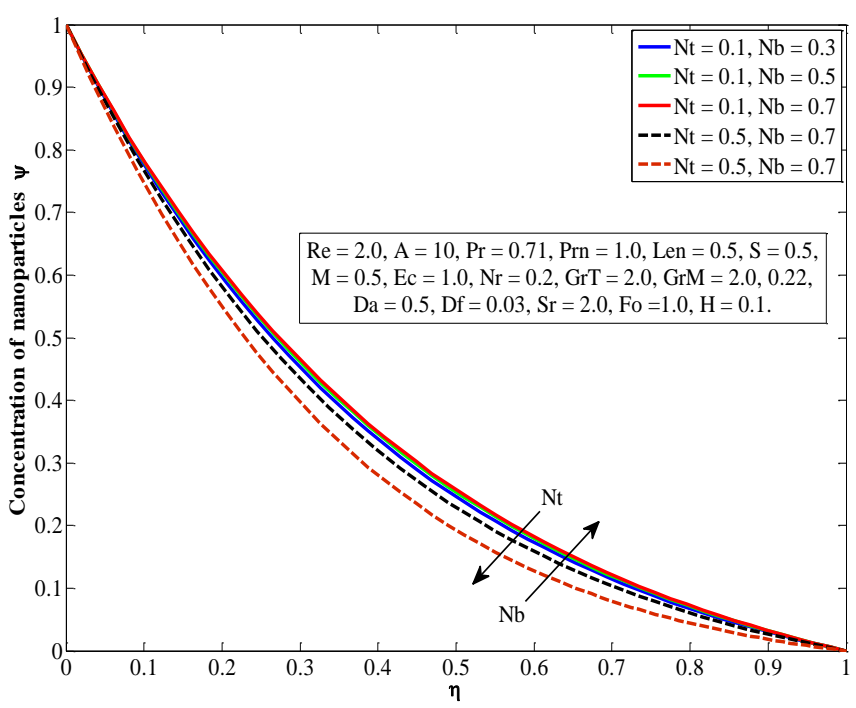

Figure 18. Concentration of nanoparticles profile for different values of $\mathrm{Nt}$ and $\mathrm{Nb}$

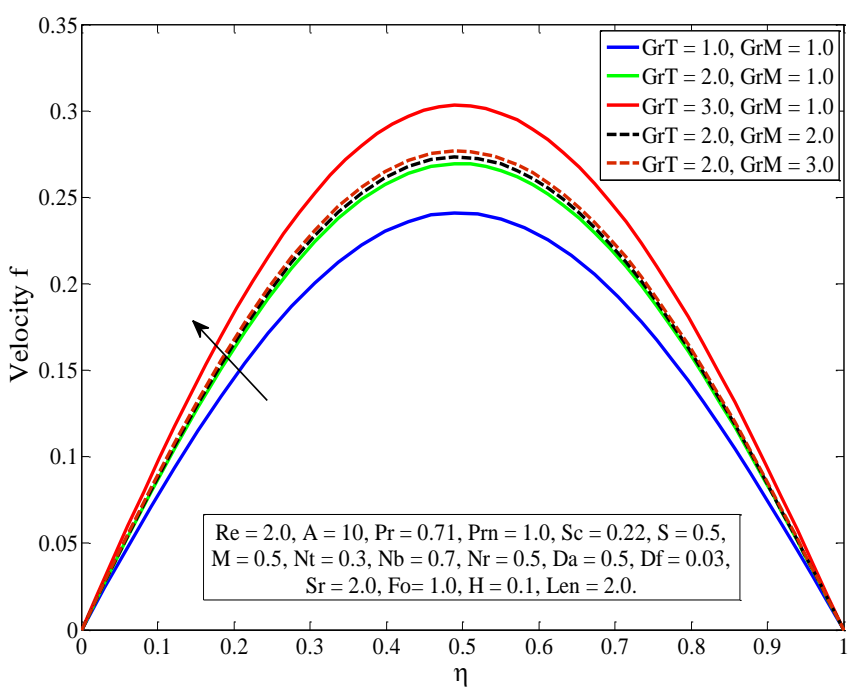

Figure 19. Velocity profile for different values of $G r_{T}$ and $G r_{M}$

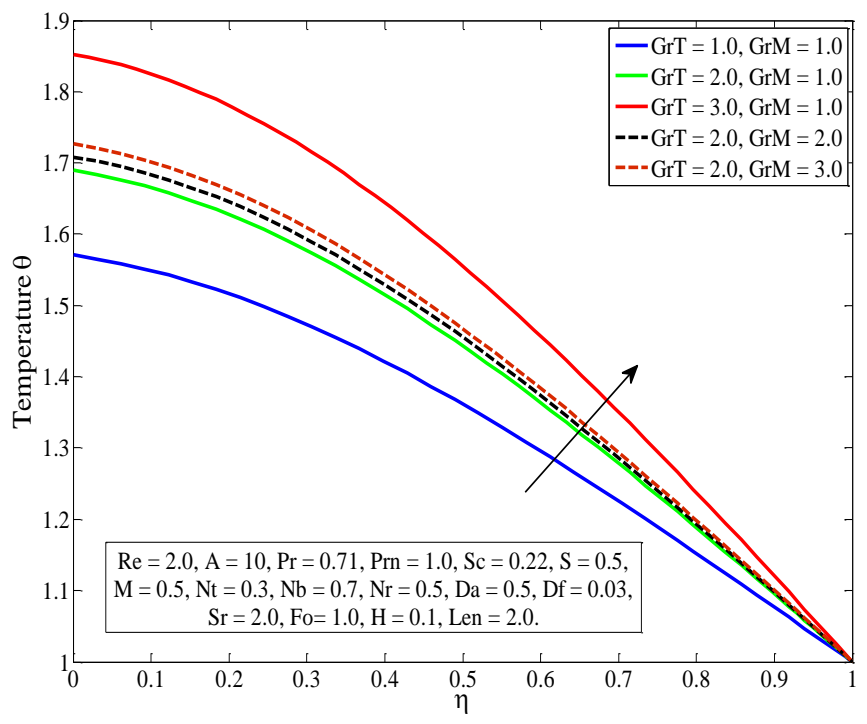

Figure 20. Temperature profile for different values of $G r_{T}$ and $G r_{M}$

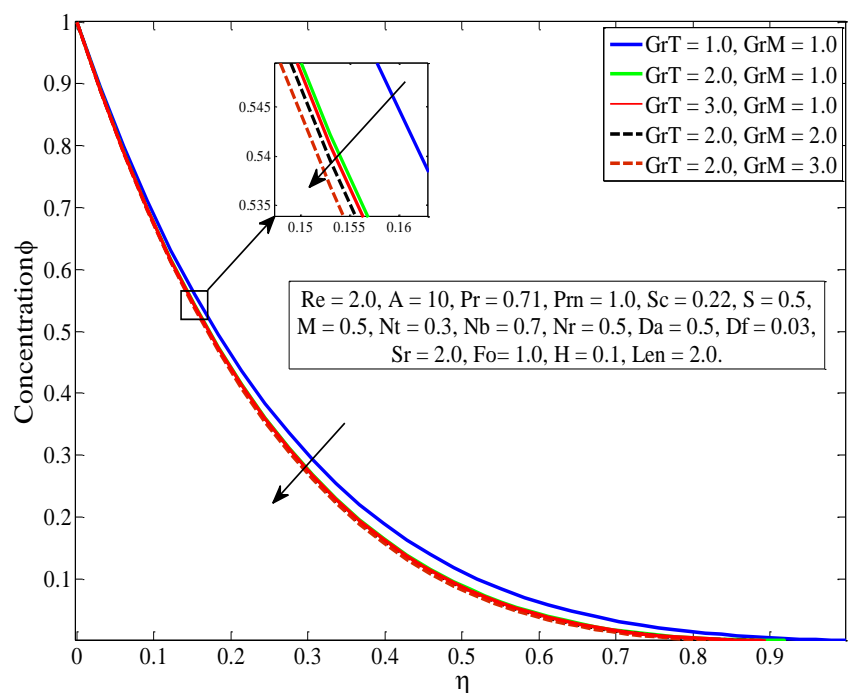

Figure 21. Concentration profile for different values of $G r_{T}$ and $G r_{M}$

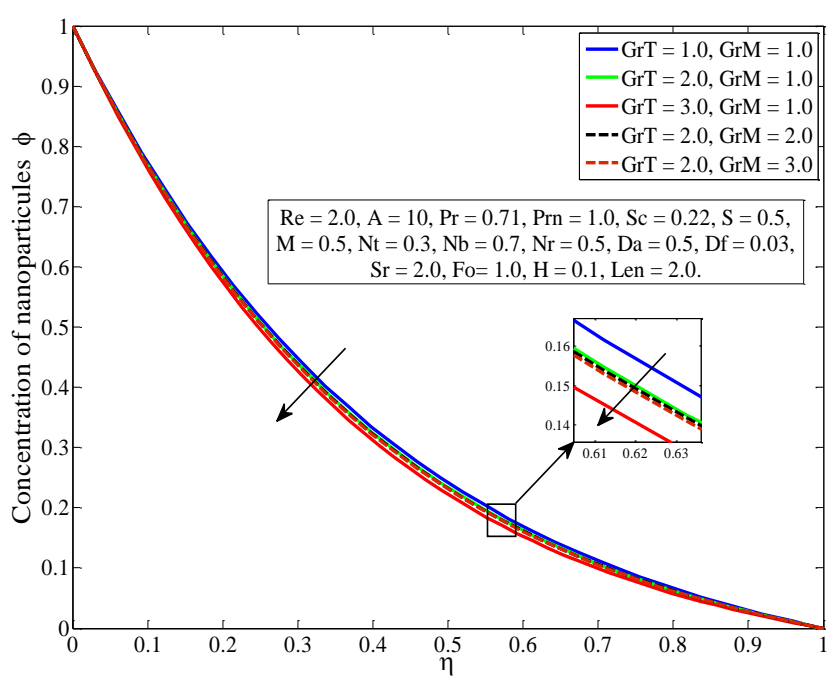

Figure 22. Concentration of nanoparticles profile for different values of $G r_{T}$ and $G r_{M}$ 
The fluid and nanoparticles concentrations for different values of $G r_{T}$, and $G r_{M}$ are displayed in the Figure 21 and 22, respectively. It is observed that by increasing $G r_{T}$, and $G r_{M}$, the fluid and the nanoparticles concentrations decelerate.

The Figure 23 demonstrates the non-dimensional velocity for different values of Nanofluid Lewis number $L e_{n}$ and the nanofluid buoyancy ratio parameter $\mathrm{N}_{\mathrm{r}}$. It is shown, from that figure, that the velocity of the fluid decreases with the increase of $\mathrm{Le}_{\mathrm{n}}$ or $\mathrm{N}_{\mathrm{r}}$.

The Figures 24 and 25 express the effects of $\mathrm{Le}_{\mathrm{n}}$ and $\mathrm{N}_{\mathrm{r}}$ on the temperature and the concentration profiles. It can be seen that as $\mathrm{Le}_{\mathrm{n}}$ increases, the temperature increases however the concentration decreases. But for $\mathrm{N}_{\mathrm{r}}$, we observed that as it increases, the temperature decreases and the concentration increases.

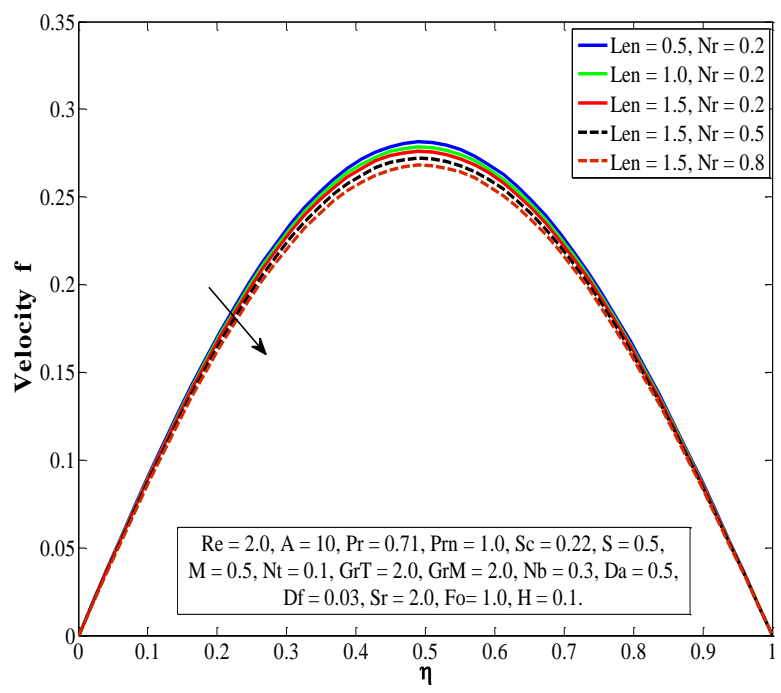

Figure 23. Velocity profile for different values of $\mathrm{Le}_{\mathrm{n}}$ and $\mathrm{N}_{\mathrm{r}}$

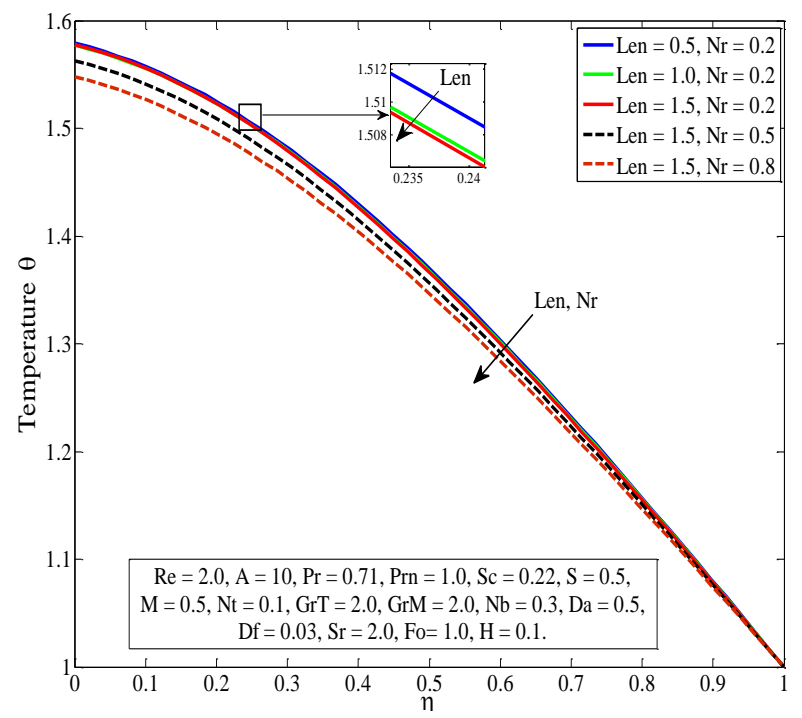

Figure 24. Temperature profile for different values of $\mathrm{Le}_{\mathrm{n}}$ and $\mathrm{N}_{\mathrm{r}}$

The Figures 24 and 25 express the effects of Nanofluid Lewis number $\mathrm{Le}_{\mathrm{n}}$ and the nanofluid buoyancy ratio parameter $\mathrm{N}_{\mathrm{r}}$ on the temperature and the concentration profiles. It can be seen that as $\mathrm{Le}_{\mathrm{n}}$ increases, the temperature increases however the concentration decreases. But for $\mathrm{N}_{\mathrm{r}}$, we observed that as it increases, the temperature decreases and the concentration increases.

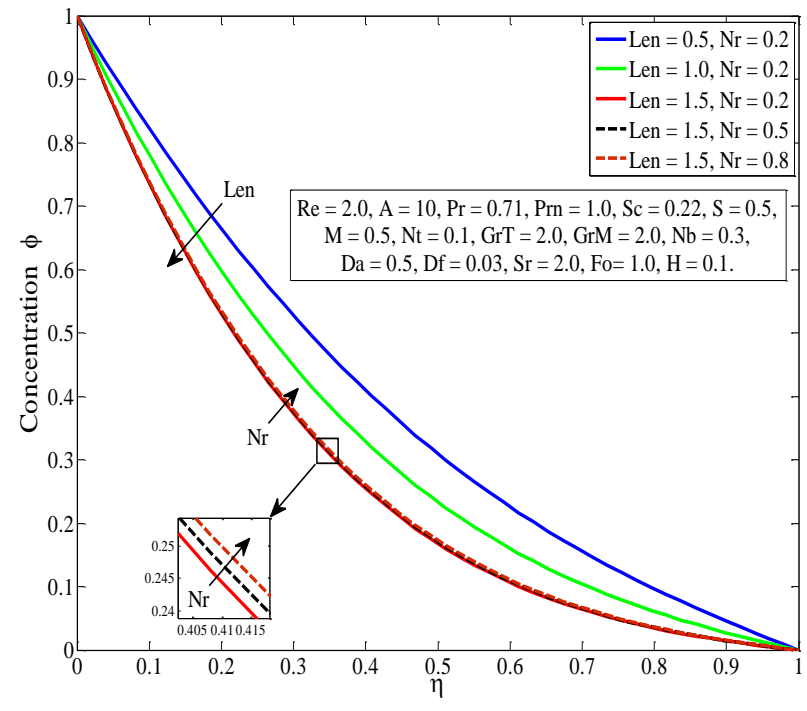

Figure 25. Concentration profile for different values of $\mathrm{Le}_{\mathrm{n}}$ and $\mathrm{N}_{\mathrm{r}}$

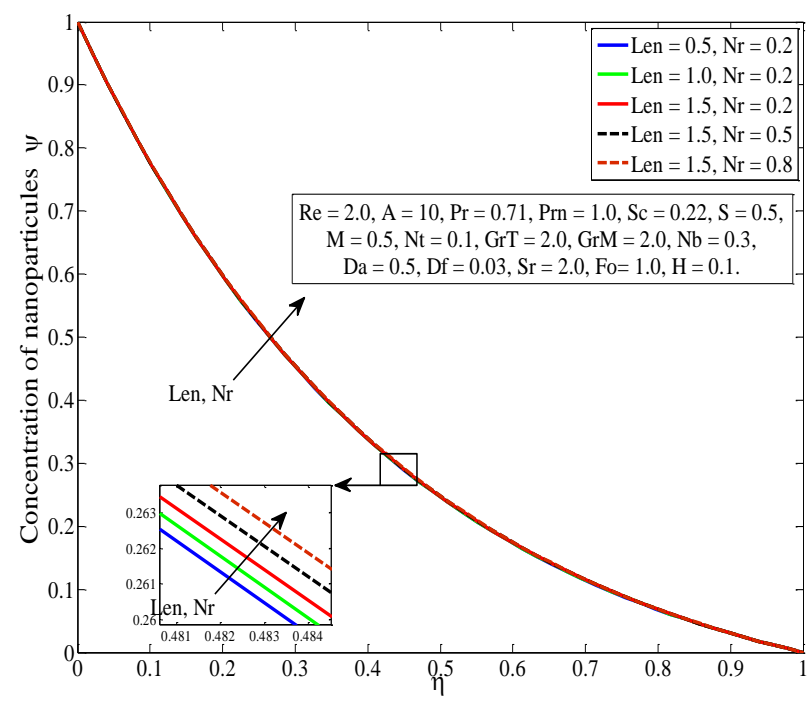

Figure 26. Concentration of nanoparticles profile for different values of $\mathrm{Le}_{\mathrm{n}}$ and $\mathrm{N}_{\mathrm{r}}$

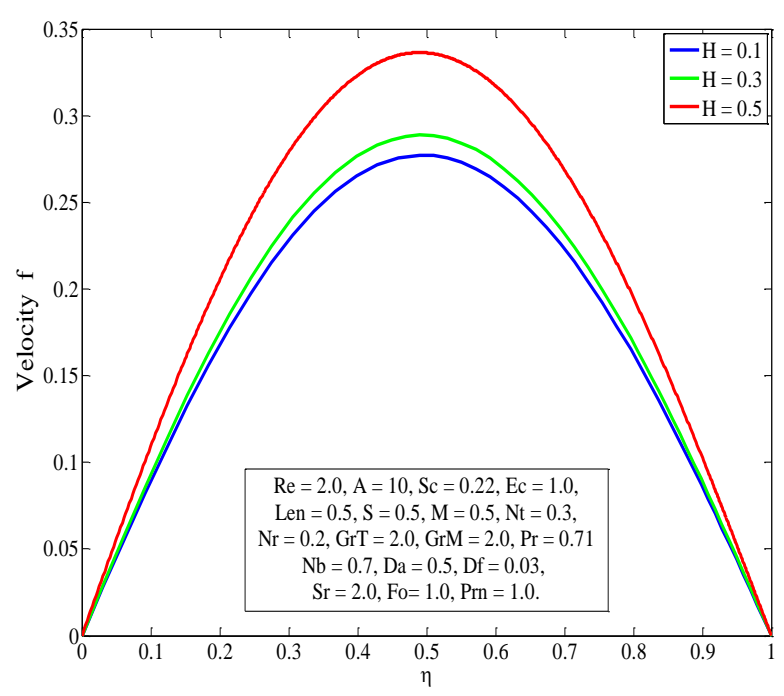

Figure 27. Velocity profile for different values of $H$

The Figure 26 shows $\mathrm{Le}_{\mathrm{n}}$ and $\mathrm{N}_{\mathrm{r}}$ effects on the concentration 
of nanoparticles profile. It is observed that the concentration of nanoparticles profile rises with increasing both of $\mathrm{Le}_{\mathrm{n}}$ and $\mathrm{N}_{\mathrm{r}}$.

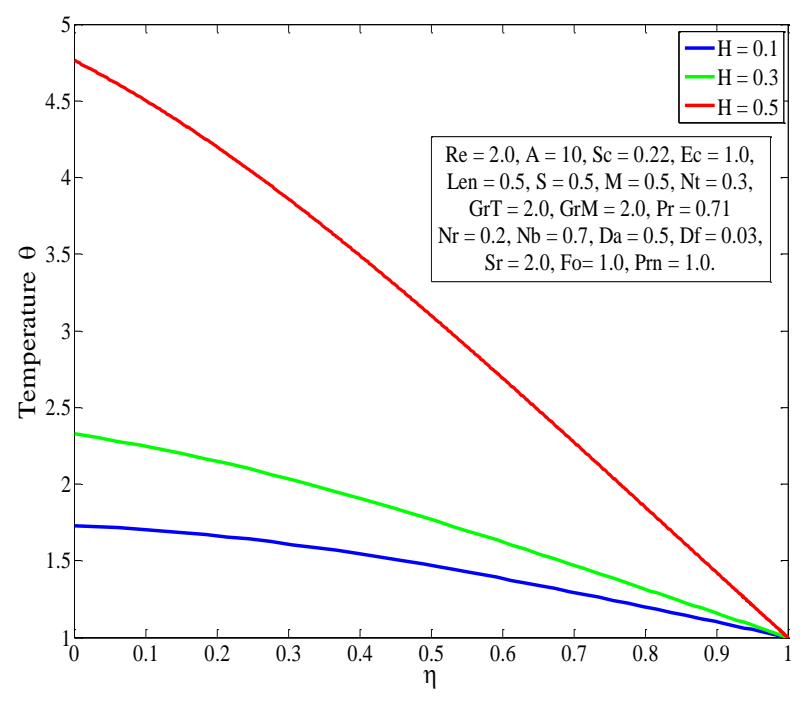

Figure 28. Temperature profile for different values of $\mathrm{H}$

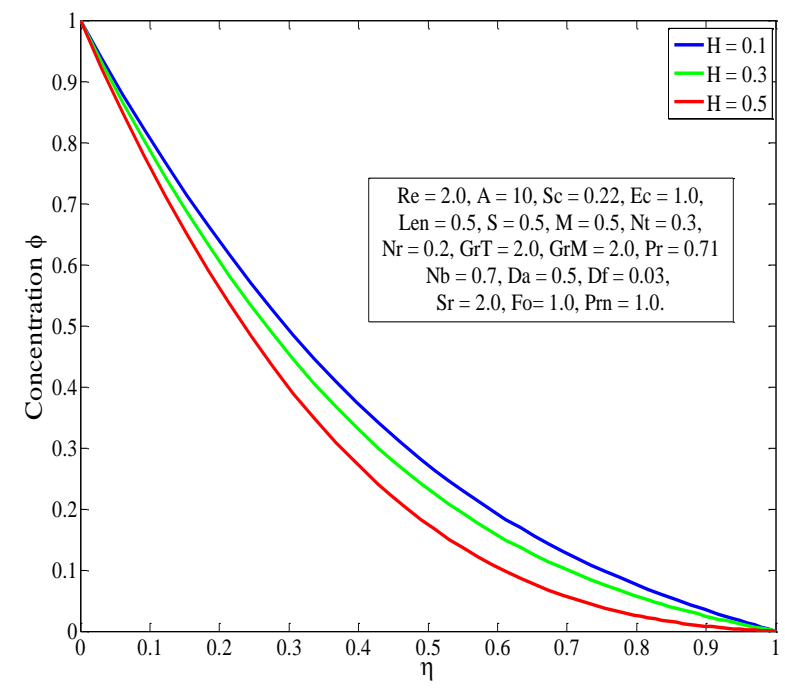

Figure 29. Concertation profile for different values of $\mathrm{H}$

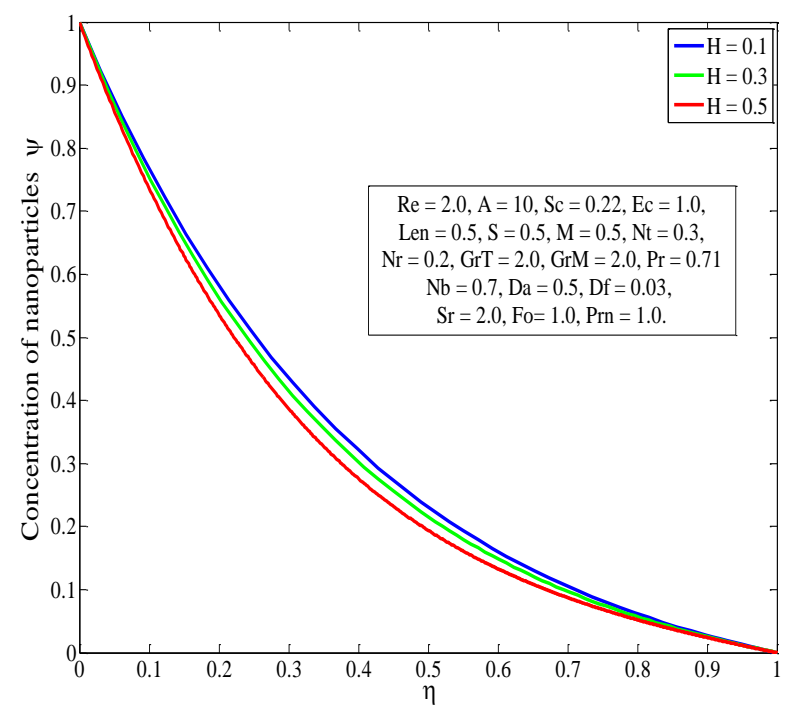

Figure 30. Concertation of nanoparticles profile for different values of $\mathrm{H}$
The Figures 27 and 28 show the non-dimensional velocity and temperature profiles for different values of the Biot number H. From these figures, it can be seen that both of velocity and temperature of the fluid increase with the raise of $\mathrm{H}$, and the augmentation of $\mathrm{H}$ causes the diminution of the fluid and the nanoparticles concentrations, as illustrated in the Figures 29 and 30.

Table 1. Values of $N u_{x}$ for the selected values of $D_{\mathrm{f}}, \mathrm{S}_{\mathrm{r}}, \mathrm{S}, \mathrm{M}$ and $\mathrm{H}$

\begin{tabular}{ccccccc}
\hline $\mathbf{D}_{\mathbf{f}}$ & $\mathbf{S}_{\mathbf{r}}$ & $\mathbf{S}$ & $\mathbf{M}$ & $\mathbf{H}$ & $\mathbf{N u}_{\mathbf{1}}$ & $\mathbf{N u}_{\mathbf{2}}$ \\
\hline $\mathbf{0 . 6 0}$ & $\mathbf{0 . 1}$ & 0.5 & 0.5 & 0.1 & 0.1702 & 0.9991 \\
$\mathbf{0 . 3 0}$ & $\mathbf{0 . 2}$ & & & & 0.1730 & 1.0297 \\
$\mathbf{0 . 1 2}$ & $\mathbf{0 . 5}$ & & & & 0.1925 & 1.2593 \\
\hline $\mathbf{0 . 6 0}$ & 0.1 & $\mathbf{0 . 5}$ & 0.5 & 0.1 & 0.1702 & 0.9991 \\
& & $\mathbf{1 . 0}$ & & & 0.1560 & 0.7689 \\
& & $\mathbf{1 . 5}$ & & & 0.1542 & 0.7393 \\
\hline 0.03 & 2.0 & 0.5 & $\mathbf{0 . 5}$ & 0.1 & 0.1702 & 0.9991 \\
& & & $\mathbf{2 . 0}$ & & 0.1690 & 0.9797 \\
& & & $\mathbf{4 . 0}$ & & 0.1675 & 0.9549 \\
\hline 0.03 & 2.0 & 0.5 & 0.5 & $\mathbf{0 . 1}$ & 0.1702 & 0.9991 \\
& & & & $\mathbf{0 . 3}$ & 0.6840 & 1.5382 \\
& & & & $\mathbf{0 . 5}$ & 1.6415 & 2.2973 \\
\hline
\end{tabular}

Table 2. Values of $S h_{x}$ for the selected values of $D_{f}, S_{r}, S, M$ and $\mathrm{H}$

\begin{tabular}{ccccccc}
\hline $\mathbf{D}_{\mathbf{f}}$ & $\mathbf{S}_{\mathbf{r}}$ & $\mathbf{S}$ & $\mathbf{M}$ & $\mathbf{H}$ & $\mathbf{S h}_{\mathbf{1}}$ & $\mathbf{S h}_{\mathbf{2}}$ \\
\hline $\mathbf{0 . 0 3}$ & $\mathbf{2 . 0}$ & 0.5 & 0.5 & 0.1 & 2.0328 & 0.3295 \\
$\mathbf{0 . 1 2}$ & $\mathbf{0 . 5}$ & & & & 2.1165 & 0.1870 \\
$\mathbf{0 . 6 0}$ & $\mathbf{0 . 1}$ & & & & 2.2606 & 0.0474 \\
\hline 0.03 & 2.0 & $\mathbf{0 . 5}$ & 0.5 & 0.1 & 2.0328 & 0.3295 \\
& & $\mathbf{1 . 0}$ & & & 1.9438 & 0.3839 \\
& & $\mathbf{1 . 5}$ & & & 1.9406 & 0.3848 \\
\hline 0.03 & 2.0 & 0.5 & $\mathbf{0 . 5}$ & 0.1 & 2.0328 & 0.3295 \\
& & & $\mathbf{2 . 0}$ & & 2.0240 & 0.3349 \\
& & & $\mathbf{4 . 0}$ & & 2.0124 & 0.3420 \\
\hline 0.03 & 2.0 & 0.5 & 0.5 & $\mathbf{0 . 1}$ & 2.0328 & 0.3295 \\
& & & & $\mathbf{0 . 3}$ & 2.2637 & 0.2358 \\
& & & & $\mathbf{0 . 5}$ & 2.5963 & 0.2461 \\
\hline
\end{tabular}

The local Nusselt and Sherwood numbers for different values of $D_{f}, S_{r}, S, M$ and $H$ for both plates are presented in the Table 1 and Table 2 respectively. The other parameters values are fixed: $\mathrm{A}=10 ; R e=G r_{T}=G r_{M}=2.0 ; P r=0.71 ; P r_{n}=$ $E c=F o=1.0 ; S c=0.22 ; D a=L e_{n}=N r=0.5 ; \mathrm{Nb}=0.7$; and $\mathrm{Nt}=0.3$.

Heat transfer rate is substantially improved with the increasing of $S_{\mathrm{r}}$ or $\mathrm{H}$. A contrary observed effect from Table 1 is with the increase of $S$ at the two walls. On the other hand, Table 1 shows that $\mathrm{M}$ is without effect on the Nusselt numbers. Interesting result is that the Dufour number plays a reverse role in relation to the Soret effect.

The mass transfer rate diminishes at the initial plate $y=-d$ and increases while this rate augments largely at the terminal $\mathrm{y}=\mathrm{d}$ when Soret number $S_{r}$ increases or Dufour number $\mathrm{D}_{\mathrm{f}}$ decreases. However, no perceptible effect is revealed with the variations of $\mathrm{S}$, the Hartmann $\mathrm{M}$ and Biot $\mathrm{H}$ numbers for high values of theses parameters.

\section{CONCLUSION}

Magnetohydrodynamic MHD flow of couple stress 
nanoparticles between two parallel walls packed with a saturated porous medium with heat and mass transfer was investigated in this study considering a great number impacting on the physical system. Similarity transformations are used for the nonlinear and highly coupled partial differential equations resulting from the complex model. The governing non-dimensional differential equations are numerically solved using the finite differences method with the Lobatto III's scheme The computational results are presented through figures respecting effects of $D_{f}, S_{r}, D a, F o$, $S, M, N b, N t, N r, L e_{n}, G r_{T}, G r_{M}, H$ on the velocity, temperature, concentration and the concentration of nanoparticles profiles..

The main observations of the present analysis are listed below:

- The Soret and Dufour effects have a great importance and cannot neglected for some specific values because they can change largely the velocity and temperature profiles. Particularly, they affect strongly the heat transfer and mass transfer rates at specific walls.

- The structural porous medium via the Darcy and Forshheimer numbers have no perceptible impact on all the profiles. This is also valuable to a couple stress nanofluid in all ranges of the Lewis number.

- The level of the couple stress fluid as well as the magnetic field imposed can substantially modified the velocity and temperature profiles but less effect on the heat and mass rates.

- The use of a nanofluid combined with a couple stress lead strictly to act on the temperature profile.

- A physical channel and thermal system with a convective heat condition at the wall is more affected compared with a system conducted by a constant temperature at the boundary.

\section{ACKNOWLEDGMENT}

This work was supported in entire part by the Biomaterials and transport phenomena laboratory agreement $\mathrm{N}^{\circ} 303$ 03-122003, at university of Medea. Mhamed Tayeb and Mohamed Najib Bouaziz acknowledge the financial support provided by DG-RSDT of Algeria.

\section{REFERENCES}

[1] Choi, S.U.S., Eastman, J.A. (1995). Enhancing thermal conductivity of fluids with nanoparticles. International Mechanical Engineering Congress and Exhibition, Conference: 1995 International Mechanical Engineering Congress and Exhibition, San Francisco.

[2] Eringen, A.C. (1966). Theory of micropolar fluids. Indiana University Mathematics Journal, 16(1): 1. http://dx.doi.org/10.1512/iumj.1967.16.16001

[3] Das, S.K., Choi, S.U., Yu, W., Pradeep, T. (2007). Nanofluids: Science and Technology. John Wiley \& Sons. https://doi.org/10.1002/9780470180693

[4] Das, S.K., Stephen, U.S. (2009). A review of heat transfer in nanofluids. Advances in Heat Transfer, 41: 81-197. https://doi.org/10.1016/S0065-2717(08)41002$\mathrm{X}$

[5] Buongiorno, J. (2006). Convective transport in nanofluids. J. Heat Transfer, 128(3): 240-250. https://doi.org/10.1115/1.2150834

[6] Stokes, V.K. (1966). Couple stresses in fluids. The $\begin{array}{llll}\text { Physics of } & \text { Fluids, } & \text { 9(9): } & 1709 .\end{array}$ https://doi.org/10.1063/1.1761925

[7] Stokes, V.K. (2012). Theories of fluids with microstructure: An introduction. Springer Science \& Business Media, Springer-Verlag Berlin Heidelberg. https://doi.org/10.1007/978-3-642-82351-0

[8] Sharma, R.C., Thakur, K.D. (2000). On couple-stress fluid heated from below in porous medium in hydromagnetics. Czechoslov. J. Phys., 50(6): 753-758. https://doi.org/10.1023/A:1022886903213

[9] Walicki, E., Walicka, A. (1999). Inertia effect in the squeeze film of a couple-stress fluid in biological bearings. Applied Mechanics and Engineering, 4(2): 363-373. https://doi.org/10.1002/tt.3020080302

[10] Vijayakumar, B., Kesavan, S. (2018). Effects of pressure distribution on parallel circular porous plates with combined effect of piezo-viscous dependency and nonNewtonian couple stress fluid. Journal of Physics: Conference $\quad$ Series, $1000(1)$ : 12021. http://dx.doi.org/10.1088/1742-6596/1000/1/012021

[11] Lin, J.R. (2017). Non-Newtonian and total inertia effects in the sphere-plate time-dependent squeeze pressure. Journal of Applied Science and Engineering, 20(2): 211-217. https://doi.org/10.6180/jase.2017.20.2.09

[12] Adesanya, S.O., Makinde, O.D. (2012). Heat transfer to magnetohydrodynamic non-Newtonian couple stress pulsatile flow between two parallel porous plates. Zeitschriftfür Naturforsch, 67(10-11): 647-656. http://dx.doi.org/10.5560/zna.2012-0073

[13] Lin, J.R., Hung, C.R. (2007). Combined effects of nonNewtonian couple stresses and fluid inertia on the squeeze film characteristics between a long cylinder and an infinite plate. Fluid Dynamics Research, 39(8): 616. http://dx.doi.org/10.1016/j.fluiddyn.2007.04.001

[14] Naduvinamani, N., Fathima, S., Hiremath, P. (2003). Hydrodynamic lubrication of rough slider bearings with couple stress fluids. Tribology International, 36(12): 949-959. https://doi.org/10.1016/S0301679X(03)00092-6

[15] Tayeb, M., Bouaziz, M.N., Hanini, S. (2019). Influence of non-linear boussinesq approximation and convective thermal boundary condition on MHD natural convection flow of a couple stress-nanofluid in a porous medium. Nano Hybrids and Composites, 26: 45-61. https://doi.org/10.4028/www.scientific.net/NHC.26.45

[16] Elsharkawy, A.A., Guedouar, L.H. (2001). An inverse solution for finite journal bearings lubricated with couple stress fluids. Tribology International, 34(2): 107-118. https://doi.org/10.1016/S0301 679X(00)00145-6

[17] Srinivasacharya, D., Kaladhar, K. (2012). Natural convection flow of a couple stress fluid between two vertical parallel plates with Hall and ion-slip effects. Acta Mechanica Sinica, 28(1): 41-50. https://doi.org/10.1007/s10409-011-0523-z

[18] Umavathi, J.C. (2011). Free convection of composite porous medium in a vertical channel. Heat Transfer Asian Research, 40(4): 308-329. https://doi.org/10.1002/htj.20340

[19] Srinivasacharya, D., Srinivasacharyulu, N., Odelu, O. (2009). Flow and heat transfer of couple stress fluid in a porous channel with expanding and contracting walls. International Communications in Heat and Mass 
Transfer,

$36(2)$ :

$180-185$

https://doi.org/10.1016/j.icheatmasstransfer.2008.10.00 5

[20] Islam, S., Zhou, C., Ran, X. (2008). Exact solutions for different vorticity functions of couple stress fluids. Journal of Zhejiang University, 9(5): 672-680. https://doi.org/10.1631/jzus.A071433

[21] Islam, S., Zhou, C.Y. (2007). Exact solutions for two dimensional flows of couple stress fluids. Zeitschrift für Angewandte Mathematik und Physik, 58(6): 1035-1048. https://doi.org/10.1007/s00033-007-5075-5

[22] Nield, D.A., Bejan, A. (2006). Convection in Porous Media. Springer, New York, NY. https://doi.org/10.1007/978-1-4757-3033-3

[23] Bujurke, N.M., Naduvinamani, N.B. (1991). On the performance of narrow porous journal bearing lubricated with couple stress fluid. Acta Mechanica, 86(1): 179-191. https://doi.org/10.1007/BF01175956

[24] Srinivasacharya, D., Kaladhar, K. (2013). Analytical solution for Hall and Ion-slip effects on mixed convection flow of couple stress fluid between parallel disks. Mathematical and Computer Modeling, 57(10): 2494-2509. https://doi.org/10.1016/j.mcm.2012.12.036

[25] Akhtar, S., Shah, N.A. (2016). Exact solutions for some unsteady flows of a couple stress fluid between parallel plates. Ain Shams Engineering Journal, 9(4): 985-992. https://doi.org/10.1016/j.asej.2016.05.008

[26] Ramesh, K. (2016). Effects of slip and convective conditions on the peristaltic flow of couple stress fluid in an asymmetric channel through porous medium. Computer Methods and Programs in Biomedicine, 135: 1-14. https://doi.org/10.1016/j.cmpb.2016.07.001

[27] Motsa, K.S., Srinivasacharya, D. (2016). Mixed convection flow of couple stress fluid in a vertical channel with radiation and Soret effects. Journal of Applied Fluid Mechanics, 9(1): 43-50. https://doi.org/10.18869/acadpub.jafm.68.224.22695

[28] Hayat, T., Asghar, S., Tanveer, A., Alsaedi, A. (2018). Chemical reaction in peristaltic motion of MHD couple stress fluid in channel with Soret and Dufour effects. Results in Physics, 10: 69-80. https://doi.org/10.1016/j.rinp.2018.04.040

[29] Srinivasacharya, D., Kaladhar, K. (2012). Mixed convection flow of couple stress fluid in a non-Darcy porous medium with Soret and Dufour effects. Journal of Engineering and Applied Science, 15(4): 415-422.

[30] Srinivasacharya, D., Kaladhar, K. (2013). Soret and Dufour effects on free convection flow of a couple stress fluid in a vertical channel with chemical reaction. Chemical Industry and Chemical Engineering Quarterly, 19(1):

45-55. http://dx.doi.org/10.2298/CICEQ111231041S

[31] Eldabe, N.T., Elshaboury, S.M., Hasan, A.A., Elogail, M.A. (2012). MHD peristaltic flow of a couple stress fluids with heat and mass transfer through a porous medium. Innovative Systems Design and Engineering, 3(5): 51-67.

[32] Makinde, O.D., Eegunjobi, A.S. (2017). MHD couple stress nanofluid flow in a permeable wall channel with entropy generation and nonlinear radiative heat. Journal of Thermal Science and Technology, 12(2): JTST0033. https://doi.org/10.1299/jtst.2017jtst0033

[33] Eegunjobi, A.S., Makinde, O.D. (2017). Irreversibility analysis of hydromagnetic flow of couple stress fluid with radiative heat in a channel filled with a porous medium. Results in Physics, 7: 459-469. https://doi.org/10.1016/j.rinp.2017.01.002

[34] Sithole, H., Mondal, H., Goqo, S., Sibanda, P., Motsa, S. (2018). Numerical simulation of couple stress nanofluid flow in magneto-porous medium with thermal radiation and a chemical reaction. Applied Mathematics and Computation, 339 : 820-836. https://doi.org/10.1016/j.amc.2018.07.042

[35] Khan, S.U., Shehzad, S.A., Rauf, A., Ali, N. (2018). Mixed convection flow of couple stress nanofluid over oscillatory stretching sheet with heat absorption/generation effects. Results in Physics, 8: 1223-1231. https://doi.org/10.1016/j.rinp.2018.01.054

\section{NOMENCLATURE}

$u, v \quad$ Velocity component along $\mathrm{x}$ and y directions, respectively.

$x, y \quad$ Cartesian coordinates along the plate and normal to it.

$T \quad$ Temperature

C Concentration

$P \quad$ Pressure

$g \quad$ Gravitational acceleration

$B_{0} \quad$ Externally imposed magnetic field in the $y$ direction

$D_{B} \quad$ Brownian diffusion coefficient

$D_{T} \quad$ Thermophoresis diffusion coefficient

$D_{m} \quad$ Molecular solutal diffusivity

$K_{T} \quad$ Thermal diffusion ratio

$T_{m} \quad$ Mean fluid temperature

$K \quad$ Porous medium parameter

$C_{S} \quad$ Concentration susceptibility

$h_{f} \quad$ Heat transfer coefficient

$f \quad$ Dimensionless velocity

$A \quad$ pressure gradient

Re Reynolds number

$S \quad$ Couple stress parameter

$G r_{T} \quad$ Thermal Grashof number

$G r_{M} \quad$ Concentration Grashof number

$\mathrm{Nr} \quad$ Nanofluid buoyancy ratio parameter

$M \quad$ Hartmann number

Da Darcy number

Fo Forchheimer number

$\mathrm{Pr} \quad$ Prandtl number

Ec Eckert number

$\mathrm{Nb} \quad$ Brownian motion parameter

$N t \quad$ thermophoresis parameter

$D_{f} \quad$ Dufour number

$S_{r} \quad$ Soret Number

$L e_{n} \quad$ Thermo-nanofluid Lewis number

$\mathrm{Pr}_{n} \quad$ Prandtl nanofluid number

$H \quad$ Biot number

$\mathrm{Nu} \quad$ Local Nusselt number

Sh Local Sherwood number

\section{Greek symbols}

$\begin{array}{ll}\phi & \text { Nanoparticles concentration } \\ \mu & \text { Dynamic viscosity } \\ \rho_{f} & \text { Density of the fluid }\end{array}$


$\rho_{P} \quad$ Density of nanoparticle

$\eta \quad$ Couple stress viscosity parameter

$\beta_{T} \quad$ Thermal expansion coefficient

$\beta_{C} \quad$ Concentration expansion coefficient

$\sigma \quad$ Electrical conductivity

$c_{f} \quad$ Forchheimer constant

$\lambda \quad$ Thermal conductivity

$\left(\rho C_{P}\right)_{f} \quad$ Heat capacity at constant pressure of fluid $\left(\rho C_{P}\right)_{P} \quad$ Heat capacity of nanoparticles

$\alpha_{f} \quad$ Thermal diffusivity

$\varepsilon \quad$ Porosity

$\eta \quad$ Similarity variable

$\theta \quad$ Dimensionless temperature

$\varphi \quad$ Dimensionless concentration

$\psi \quad$ Dimensionless concentration of nanoparticles 